\title{
Preliminary results on the seroprevalence of Angiostrongylus vasorum and co-infection with Dirofilaria immitis in shelter dogs from Portugal
}

\author{
AM Alho $^{1 *}$, M Schnyder $^{2}$, J Meireles ${ }^{1}$, S Belo ${ }^{3}$, P Deplazes² $^{2}$, L Madeira de Carvalho $^{1}$ \\ From The 1st Conference on Neglected Vectors and Vector-Borne Diseases (EurNegVec): with Management \\ Committee and Working Group Meetings of the COST Action TD1303 \\ Cluj-Napoca, Romania. 8-11 April 2014
}

Angiostrongylosis and dirofilariosis have recently revived the attention of the scientific community due to their emergence in several geographical areas. Angiostrongylus vasorum is responsible for verminous pneumonia and also for neurological, cardiovascular and coagulation disorders while Dirofilaria immitis is responsible for right side heart failure. Portugal is an endemic country for $D$. immitis. However, prevalence data concerning A. vasorum is scarce. Considering that documented cross-reactions between these parasites may lead to incorrect or misfit diagnoses, we aimed to assess the prevalence and potential co-infections of both canine heartworms.

An epidemiological survey was conducted, involving 341 sera collected from shelter dogs housed in three coastal districts of Portugal: Coimbra, Santarém and Setúbal. Sera were tested for circulating A. vasorum antigens by a sandwich-ELISA using mono and polyclonal antibodies (sensitivity $95.7 \%$, specificity $94.0 \%$, Schnyder et al., 2011) and for specific antibodies against purified $A$. vasorum adult stage antigen (sensitivity $85.7 \%$, specificity $98.8 \%$, Schucan et al. 2012). In order to detect the presence of $D$. immitis circulating antigens, a commercial kit WITNESS ${ }^{\circledR}$ Dirofilaria (Synbiotics Corp., Europe, sensitivity $100 \%$ and specificity $98.3 \%$ ) based on rapid immunomigration technology was used. Statistical analysis was performed with SPSS program and estimated prevalence was obtained using Agresti-Coull method.

Regarding A. vasorum, a total of $1.17 \%$ (4/341, 95\% Confidence Intervals, CI: $0.3-3.1 \%)$ of the animals were positive in both ELISAs, while 1.76\% (6/341, CI: 0.7-3.9\%) were

\footnotetext{
* Correspondence: admargaridaalho@fmv.ulisboa.pt

'Centro de Investigação Interdisciplinar em Sanidade Animal, Faculdade de Medicina Veterinária, Universidade de Lisboa, Portugal

Full list of author information is available at the end of the article
}

only antigen-positive and $2.35 \%$ (8/341, CI: $1.1-4.6 \%)$ only antibody-positive. Positive dogs were from the three surveyed areas. Concerning D. immitis, a total of $7.92 \%$ of the animals were positive (27/341, CI: $5.5-11.3 \%)$, also from the three areas. Out of the 341 sera analysed, one dog was positive for $D$. immitis circulating antigen and A. vasorum antibody-positive only.

These results show a slightly higher seroprevalence of angiostrongylosis in Portugal in comparison with seroprevalence found in UK, Italy, Germany and Poland, probably explained by the fact that this study was performed with shelter dogs, usually not under prophylaxis and therefore at a higher risk of infection.

To the authors' knowledge, this is the first study performed in Portugal to assess A. vasorum seroprevalence in dogs and $D$. immitis co-infection. We expect that the obtained data will enhance the awareness of veterinary practitioners concerning these diseases and reinforce the importance of a more targeted preventive therapy for heartworms in companion animals in Portugal.

\section{Funding}

PhD research grant SFRH/BD/85427/2012; Project PTDC/ SAU-SAP/113523/2009 supported by Fundação para a Ciência e a Tecnologia (FCT), Portugal.

\footnotetext{
Authors' details

${ }^{1}$ Centro de Investigação Interdisciplinar em Sanidade Animal, Faculdade de Medicina Veterinária, Universidade de Lisboa, Portugal. ${ }^{2}$ Institute of Parasitology, Vetsuisse Faculty, University of Zurich, Switzerland. ${ }^{3}$ Unidade de Parasitologia Médica, Instituto de Higiene e Medicina Tropical, Universidade Nova de Lisboa, Portugal.
} 
Submit your next manuscript to BioMed Central and take full advantage of:

- Convenient online submission

- Thorough peer review

- No space constraints or color figure charges

- Immediate publication on acceptance

- Inclusion in PubMed, CAS, Scopus and Google Scholar

- Research which is freely available for redistribution

Submit your manuscript at www.biomedcentral.com/submit
C Biomed Central 